\title{
Visible Angle on Magnetic Resonance Imaging Can Be Considered Indicator of Postoperative Outcome in Retropubic Radical Prostatectomy
}

\author{
Se Yun Kwon ${ }^{1}$, Jun Nyung Lee ${ }^{2}$, Yun-Sok $\mathrm{Ha}^{2}$, Seock Hwan Choi ${ }^{2}$, \\ Hyun Tae Kim², Tae-Hwan Kim², Tae Gyun Kwon ${ }^{2}$ \\ ${ }^{1}$ Department of Urology, Dongguk University College of Medicine, Gyeongju, Korea \\ ${ }^{2}$ Department of Urology, Kyungpook National University School of Medicine, Daegu, Korea
}

\begin{abstract}
Purpose: We aimed to measure the visible angle (VA) outside the surgical field of view using preoperative magnetic resonance imaging (MRI), and to relate structural data to clinical outcome.

Materials and Methods: We retrospectively analyzed data obtained from 322 consecutive patients who underwent robot-assisted laparoscopic radical prostatectomy (RALP) or radical retropubic prostatectomy (RRP) for prostate cancer from January 2011 to June 2013, with at least 1 year of follow-up. Patients were divided into 4 groups, according to median VA values and surgical approach. VA was defined as the angle between the horizontal line on view, rotated to $90^{\circ}$ from the midsagittal T2-weighted sequence image, and the tangent line of the highest protruding surface of the symphysis pubis.

Results: The larger VA was, the shorter mean operative time (213.2 minutes vs. 174.2 minutes, $p=0.002$ ) was significantly and the smaller VA was, the higher complication rate $(10.8 \%$ vs. $1.6 \%, p=0.043)$ was in RRP patients. There were no significant differences in operative outcomes in RALP patients.

Conclusions: VA in midsagittal T2-weighted sequence predict operative time and complication rates in retropubic radical prostatectomy, and thus, the value may be used as surgical indications for retropubic radical prostatectomy. (Korean J Urol Oncol 2017;15:38-43)
\end{abstract}

Key Words: Robot-assisted laparoscopic radical prostatectomy $\cdot$ Retropubic radical prostatectomy $\cdot$ Magnetic resonance imaging

\section{INTRODUCTION}

Open retropubic radical prostatectomy (RRP) is the gold standard treatment for localized prostate cancer. ${ }^{1,2}$ However, RRP is a skillful surgery because of a narrow surgical field and complex anatomy. Recently, robot-assisted laparoscopic radical

Received March 9, 2017, Revised April 1, 2017,

Accepted April 6, 2017

Corresponding Author: Tae-Hwan Kim

Department of Urology, Kyungpook National University School of Medicine, 680 Gukchaebosang-ro, Jung-gu, Daegu 41944, Korea

E-mail: doctork@knu.ac.kr

Tel: +82-53-200-2166, Fax: +82-53-421-9618 prostatectomy (RALP) has been applied to overcome these problems.

According to a recent study, RALP demonstrated better functional operative outcomes in terms of urinary incontinence, potency, postoperative pain, intraoperative blood loss, and length of hospital stay compared with RRP. ${ }^{3-7}$ In addition, oncologic outcomes following RALP were similar to those reported with RRP. ${ }^{5,7,8}$

However, RRP generally demonstrated better outcomes than RALP with respect to the operative time. ${ }^{9}$ In fact, operative time has become an important factor when deciding on which operative method to use for prostate cancer patients.

(i) (1) This is an Open Access article distributed under the terms of the Creative Commons Attribution Non-Commercial License (http://creativecommons.org/licenses/by-nc/4.0/) which permits unrestricted non-commercial use, distribution, and reproduction in any medium, provided the original work is properly cited. 2017 (C) Copyright The Korean Urological Oncology Society and The Korean Prostate Society. All Rights Reserved. 
We analyzed factors affecting operative outcomes in each operative method. In particular, we noticed that pelvic anatomic structure affected operative outcomes; we therefore measured the visible angle (VA) that exceeded the field of vision by using preoperative magnetic resonance imaging (MRI). We divided patients into groups according to the surgical approach, subdivided them according to the VA and compared the operative outcomes of each group.

\section{MATERIALS AND METHODS}

\section{Patients}

We retrospectively analyzed data obtained from 322 consecutive patients who underwent RALP and RRP for prostate cancer between January 2011 and June 2013, and who had at least 1 year of follow-up. Institutional review board approval was obtained before data retrieval and analysis (KNUMc 13-1060). Study participants were consent to publication of material about them. All patients underwent RALP or RRP performed by a single experienced surgeon who had performed over 200 RALP and 500 RRP procedures. All patients who underwent RRP were divided into groups $\mathrm{A}$ and $\mathrm{B}$ according to the median value of the VA, which was $125^{\circ}$. Patients with a VA of $<125^{\circ}$ were defined as group $\mathrm{A}$ and those with a VA of $\geq 125^{\circ}$ were

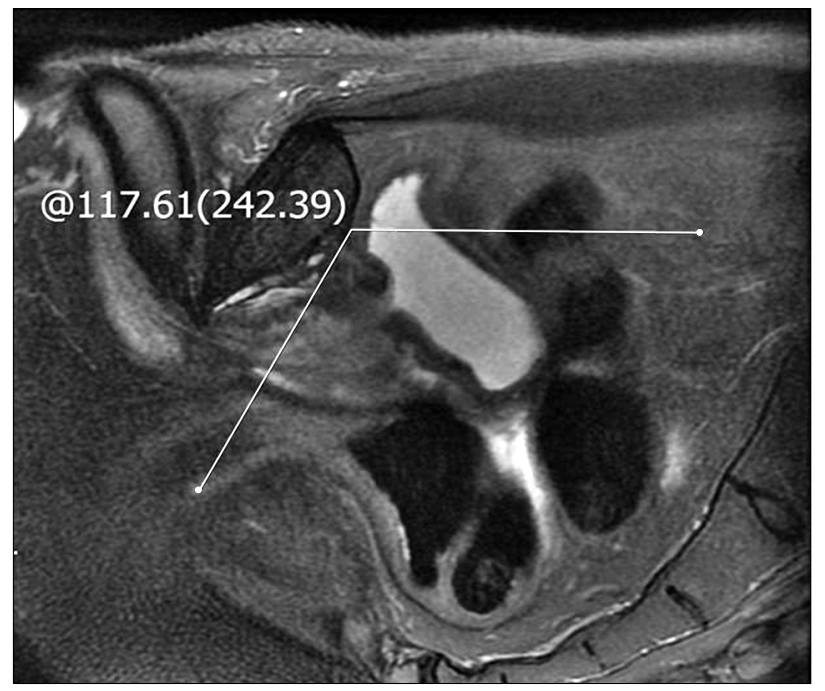

Fig. 1. Visible angle on a rotated $90^{\circ}$ view of a midsagittal T2-weighted image (groups A and C). RRP: radical retropubic prostatectomy, RALP: robot-assisted laparoscopic prostatectomy. Group A: patients who underwent RRP with a visible angle (VA) of $<125^{\circ}$, group $\mathrm{C}$ : patients who underwent RALP with a VA of $<125^{\circ}$. defined as group B. All patients who underwent RALP were similarly divided into groups $\mathrm{C}$ and $\mathrm{D}$ (Figs. 1, 2).

\section{Definition of VA}

All MRI scans in our study were retrospectively interpreted by a single radiologist, and all clinical, pathological, and functional findings were blinded. The VA was defined as the angle between the horizontal line and tangent line of the most protruding posterior part in the symphysis pubis, observed on a rotated $90^{\circ}$ view of a mid-sagittal T2-weighted image.

\section{Surgical Technique}

The RALP procedure included the use of a 3-arm da Vinci robot (Intuitive Surgical, Sunnyvale, CA, USA). The 6-port transperitoneal technique was performed for all patients. In short, patients were placed in the Trendelenburg position at the standard $30^{\circ}$ angle. We coagulated the superficial dorsal vein and removed the preprostatic fat. Using a Bovie knife, the bladder-prostate junction was divided along the bladder-prostate imaginary borderline until the prostatic urethra was exposed. The prostatic urethra was incised and a previously placed Foley urinary catheter was inspected. We continued to divide the bladder and prostate in the remaining regions until the prostate was completely divided. The seminal vesicle was exposed, the periseminal vesicle was dissected and ligated, and the vas deferens

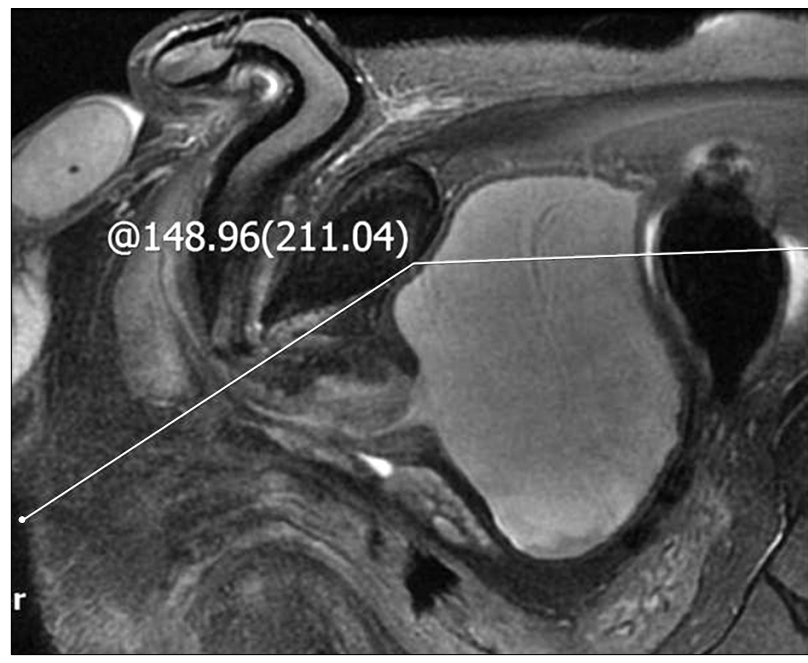

Fig. 2. Visible angle on a rotated $90^{\circ}$ view of a midsagittal T2-weighted image (groups B and D). RRP: radical retropubic prostatectomy, RALP: robot-assisted laparoscopic prostatectomy. Group B, patients who underwent RRP with a visible angle (VA) $\geq 125^{\circ}$, group $\mathrm{D}$, patients who underwent RALP with a VA $\geq 125^{\circ}$. 
was divided. We ligated most of the vascular pedicles with 5-mm titanium ligation clips (Aesculap AG, a subsidiary of B. Braun Melsungen AG, Tuttlingen, Germany). Large diameter vessels, which were unsuitable for ligation with the titanium clips, were ligated using Hem-o-lok clips (Teleex Medical, Durham, NC, USA). Denonvillier's fascia was incised and the posterolateral surface of the prostate was subsequently dissected caudally on each side by using scissors. Neurovascular bundles on both sides of the prostate gland were saved by using the interfascial technique. When adherence was severe and tumor invasion could not be determined with certainty, we dissected a wider plane for oncological safety. The prostatic apex was cut as distally as possible, leaving a cuff anastomosed to the bladder neck. The deep dorsal vein complex was ligated by suturing with a 3.0 Monocyn (Aesculap AG) after positioning the prostate in an Endopouch. After posterior prostatic musculofascial reconstruction, the bladder neck and the distal urethral stump were continuously anastomosed using a 3.0 Monocyn suture. The previously placed $16 \mathrm{~F}$ Foley urinary catheter was retained. The catheter was placed and the bladder was filled with $120 \mathrm{~mL}$ of normal saline to examine if any leakage occurred. The catheter was left in place for 7 days.

During RRP, we dissected the bladder and most importantly, the prostate junction, in exactly the same way as in RALP. After dissection of the vas deferens and seminal vesicle, the posterolateral surface of the prostate was dissected in an antegrade direction, including bilateral nerve sparing. Finally, the urethra was cut at the prostatic apex. After careful hemostasis, a Rocco suture was applied. Subsequently, continuous urethrovesical anastomosis was performed, similar to that performed in RALP.

\section{Definition and Assessment of Continence}

Continence was defined as using no pads and having no urine leakages, as determined by patient responses. Patients were asked the following question: "How many pads or adult diapers did you use per day to control leakage during the past 4 weeks?" Recovery of continence was evaluated routinely at $1,3,6$, and 12 months after surgery.

\section{Statistical Analysis}

Demographics and perioperative outcomes were analyzed by using the chi-square test and Student t-test. The chi-square test was used to analyze continence rates at the above mentioned times. Multivariate analysis was performed through logistic regression analysis. Statistical analyses were performed by using IBM SPSS Statistics ver. 20.0 (IBM Co., Armonk, NY, USA). For all statistical comparisons, $\mathrm{p}$-values of $<0.05$ were considered statistically significant.

\section{RESULTS}

Groups A and B included 37 and 62 patients, respectively, and groups $\mathrm{C}$ and D included 130 and 93 patients, respectively. There were no significant differences in the age, body mass index (BMI), prostate volume, preoperative prostate-specific antigen level, pathologic Gleason score, and pathologic stage between groups $\mathrm{A}$ and $\mathrm{B}$, or between groups $\mathrm{C}$ and $\mathrm{D}$ (Table 1). The mean operative time was significantly longer in group $\mathrm{A}$ than in group B (213.2 minutes vs. 174.2 minutes, $\mathrm{p}=0.002)$. There was no significant difference in the estimated blood loss between groups A and B (227.9 mL vs. $268.6 \mathrm{~mL}, \mathrm{p}=0.209)$. The complication rate was higher in group $\mathrm{A}$ than in group $\mathrm{B}$ (10.8\% vs. $1.6 \%, \mathrm{p}=0.043$ ). According to the Clavien-Dindo classification, ${ }^{10}$ In group A, 1 complication was grade I and 3 complications were grade II and grade I case is atelectasis and grade II were postoperative bleeding. In group B, 1 complication was grade II and it was wound dehiscence. The mean operative time was similar in groups C and D (249.6 minutes vs. 237.7 minutes, $\mathrm{p}=0.144$ ). There was no significant difference in the estimated blood loss between groups C and D (196.1 $\mathrm{mL}$ vs. $187.7 \mathrm{~mL}, \mathrm{p}=0.582$ ). The complication rate did not differ statistically between groups $\mathrm{C}$ and $\mathrm{D}(3.1 \%$ vs. $2.2 \%$, $\mathrm{p}=0.673$ ). In group C, 2 complications were grade I and 2 complications were grade II and grade I case is atelectasis and grade II were postoperative bleeding and ileus. In group D, 2 complications were grade II and they were pneumonia and ileus. Urine leakage was similar in groups A and B (16.2\% vs. $11.3 \%, \mathrm{p}=0.483)$ and groups $\mathrm{C}$ and $\mathrm{D}(8.5 \%$ vs. $4.3 \%, \mathrm{p}=0.221)$ (Table 2).

The continence rate was not significantly different in groups $\mathrm{A}$ and $\mathrm{B}$ or in groups $\mathrm{C}$ and $\mathrm{D}$, as classified according to the VA. At 12 months, groups A, B, C, and D had incontinence rates of $18.9 \%, 14.5 \%, 10.0 \%$, and $7.5 \%$, respectively.

The surgical positive margin rate was not significantly different among all the groups compared. Groups A and B had surgical positive margin rates of $24.3 \%$ and $33.9 \%$, respectively ( $p=0.317$ ), and groups $C$ and $D$ had rates of $34.6 \%$ and $40.9 \%$, 
Table 1. Comparison of demographic data, perioperative surgical outcomes, and pathologic data

\begin{tabular}{|c|c|c|c|c|c|c|}
\hline \multirow{2}{*}{ Variable } & \multicolumn{3}{|c|}{ RRP } & \multicolumn{3}{|c|}{ RALP } \\
\hline & Group A $(n=37)$ & Group B $(n=62)$ & p-value & Group C $(n=130)$ & Group D $(n=93)$ & p-value \\
\hline Age $(y r)$ & $65.8 \pm 5.7$ & $65.3 \pm 5.6$ & 0.647 & $64.7 \pm 6.2$ & $65.5 \pm 6.9$ & 0.377 \\
\hline Body mass index $\left(\mathrm{kg} / \mathrm{m}^{2}\right)$ & $24.1 \pm 2.3$ & $23.3 \pm 2.6$ & 0.171 & $24.2 \pm 3.0$ & $23.6 \pm 2.6$ & 0.137 \\
\hline Prostate volume $(\mathrm{mL})$ & $38.9 \pm 18.3$ & $37.0 \pm 13.0$ & 0.545 & $36.9 \pm 18.6$ & $37.1 \pm 15.3$ & 0.912 \\
\hline Preoperative PSA (ng/mL) & & & 0.262 & & & 0.718 \\
\hline$<10$ & 17 & 36 & & 78 & 58 & \\
\hline $10-20$ & 9 & 16 & & 38 & 23 & \\
\hline$>20$ & 11 & 10 & & 14 & 12 & \\
\hline Pathologic Gleason score & & & 0.221 & & & 0.768 \\
\hline 6 & 8 & 12 & & 24 & 11 & \\
\hline 7 & 18 & 41 & & 78 & 62 & \\
\hline 8 & 7 & 7 & & 17 & 11 & \\
\hline 9 & 4 & 2 & & 9 & 8 & \\
\hline 10 & 0 & 0 & & 2 & 1 & \\
\hline Pathologic stage & & & 0.589 & & & 0.376 \\
\hline $\mathrm{T} 2$ & 23 & 38 & & 81 & 48 & \\
\hline T3a & 11 & 15 & & 35 & 30 & \\
\hline $\mathrm{T} 3 \mathrm{~b}$ & 3 & 9 & & 13 & 13 & \\
\hline $\mathrm{T} 4$ & 0 & 0 & & 1 & 2 & \\
\hline
\end{tabular}

Values are presented as mean \pm standard deviation or number.

All patients who underwent RRP with a visible angele (VA) of $<125^{\circ}$ were defined as group A and those with a VA of $\geq 125^{\circ}$ were defined as group B. All patients who underwent RALP with VA of $<125^{\circ}$ were defined as group $\mathrm{C}$ and those with a VA of $\geq 125^{\circ}$ were defined as group D.

RRP: radical retropubic prostatectomy, RALP: robot-assisted laparoscopic prostatectomy, PSA: prostate-specific antigen.

Table 2. Comparison of demographic data, perioperative surgical outcomes and pathologic data

\begin{tabular}{|c|c|c|c|c|c|c|}
\hline \multirow{2}{*}{ Variable } & \multicolumn{3}{|c|}{ RRP } & \multicolumn{3}{|c|}{ RALP } \\
\hline & Group A $(n=37)$ & Group B $(n=62)$ & p-value & Group C $(n=130)$ & Group D $(n=93)$ & $\mathrm{p}$-value \\
\hline Operative time (min) & $213.2 \pm 51.3$ & $174.2 \pm 64.0$ & 0.002 & $249.6 \pm 60.4$ & $237.7 \pm 59.0$ & 0.144 \\
\hline Estimated blood loss (mL) & $227.9 \pm 190.1$ & $268.6 \pm 130.0$ & 0.209 & $196.1 \pm 122.2$ & $187.7 \pm 97.8$ & 0.582 \\
\hline Complication (\%) & $4(10.8)$ & $1(1.6)$ & 0.043 & $4(3.1)$ & $2(2.2)$ & 0.673 \\
\hline Urine leakage $(\%)$ & $6(16.2)$ & $7(11.3)$ & 0.483 & $11(8.5)$ & $4(4.3)$ & 0.221 \\
\hline \multicolumn{7}{|l|}{ PPI $(\%)$} \\
\hline 1 Month & $30(81.1)$ & $46(74.2)$ & 0.432 & $65(50.0)$ & $60(64.5)$ & 0.986 \\
\hline 3 Months & $28(75.7)$ & $39(62.9)$ & 0.267 & $69(53.1)$ & $50(53.8)$ & 0.919 \\
\hline 6 Months & $18(48.6)$ & $29(46.8)$ & 0.857 & $51(39.2)$ & $37(39.8)$ & 0.933 \\
\hline 12 Months & $7(18.9)$ & $9(14.5)$ & 0.565 & $13(10.0)$ & $7(7.5)$ & 0.524 \\
\hline Positive surgical margin (\%) & $9(24.3)$ & $21(33.9)$ & 0.317 & $45(34.6)$ & $38(40.9)$ & 0.341 \\
\hline
\end{tabular}

Values are presented as mean \pm standard deviation or number $(\%)$.

All patients who underwent RRP with a visible angele (VA) of $<125^{\circ}$ were defined as group A and those with a VA of $\geq 125^{\circ}$ were defined as group B. All patients who underwent RALP with VA of $<125^{\circ}$ were defined as group C and those with a VA of $\geq 125^{\circ}$ were defined as group D.

RRP: radical retropubic prostatectomy, RALP: robot-assisted laparoscopic prostatectomy, PPI: post prostatectomy incontinence.

respectively $(\mathrm{p}=0.341)$.

On multivariate analysis of various factors, prostate volume, BMI, operative type and VA proved to be independently asso- ciated with the operative time (Table 3). However, for complication, none of the factors demonstrated significant associations on multivariate analysis. 
Table 3. Multivariate analysis of various factors potentially affecting operative time

\begin{tabular}{lcc}
\hline \multicolumn{1}{c}{ Variable } & OR $(95 \% \mathrm{CI})$ & $\mathrm{p}$-value \\
\hline Patient age & $1.010(0.970-1.053)$ & 0.621 \\
Prostate volume & $1.016(0.999-1.032)$ & 0.038 \\
Body mass index & $1.039(0.947-1.139)$ & 0.021 \\
Preoperative PSA & $1.032(1.008-1.057)$ & 0.110 \\
Operative type & $1.147(1.080-1.269)$ & 0.001 \\
Pathologic stage & $1.192(0.809-1.756)$ & 0.376 \\
Pathologic Gleason sum & $1.116(0.971-1.021)$ & 0.123 \\
Visible angle & $1.611(1.345-1.083)$ & 0.042 \\
\hline
\end{tabular}

OR: odd ratio, CI: confidence interval, PSA: prostate-specific antigen.

\section{DISCUSSION}

Since RALP was introduced, several studies have reported the short- and long-term operative outcomes compared with $\mathrm{RRP}^{3-8}$ RALP has been known to facilitate the preservation of neuromuscular structures and help prevent complications such as erectile dysfunction and urinary incontinence. Moreover, RALP has the benefits associated with laparoscopic surgery such as lower intraoperative bleeding, less postoperative pain, and shorter surgical wounds. However, disadvantages of RALP include longer operative times and higher costs.

Although there are numerous publications comparing the results of RALP with RRP in terms of the surgical outcomes, there is no clear indication about which surgical approach is better.

Herein, we monitored the results obtained by using preoperative MRI performed for staging. MRI is an established, noninvasive tool for studying pelvic anatomy. Several studies regarding the use of MRI for monitoring purposes have been reported. For example, Sohn et al. ${ }^{11}$ evaluated the changes in pelvic floor anatomy after RRP by using MRI before and after continence recovery.

Lim et al. ${ }^{12}$ assessed the factor associated with recovery from urinary incontinence after RRP. Membranous urethral length and shape measured by preoperative MRI were associated with a return of continence at 12 months. Matikainen et al. ${ }^{13}$ reported that the depth of the prostatic apex measured with preoperative MRI is an independent predictor of positive apical margins at RRP. However, there have been no studies reporting the relationship between the preoperative MRI findings and operative indications.

RRP procedures such as ligation of the dorsal vein complex, nerve sparing, and vesicourethral anastomosis are very complex; therefore performing RRP in a narrow pelvic space is often difficult for urology surgeons and many surgeons have faced difficulties in complete exposure of prostate and especially, exposure in apical portion of prostate due to its anatomical position. We paid attention to VA that is formed surgical incisional line and surgeon's visual field. In our experience, the smaller visual angle, the more difficult operation we filled. Moreover, a protruding state of the pubic symphysis is an important anatomic landmark for securing the operative field and it affects the VA with respect to the posterior slope of the pubic bone. When the pubic symphysis is not protruded, the VA is lager and the secured operative visual field is wider. However, in the case of a severely protruding pubic symphysis, the VA is not similar to the posterior slope of the pubic bone. Specifically, the VA is smaller and the view of the operative field becomes narrower. Therefore, we analyzed that the VA could affect the operative outcomes.

We divided patients who underwent RRP on the basis of the median value of the VA. Matikainen et al. ${ }^{13}$ analyzed median value of pelvic dimension, interspinous distance and apical depth to expect operative outcome of RRP and Neill et al. ${ }^{14}$ evaluated median value various computed tomographic pelvimetry to predict technical difficulties during open RRP.

In patients who underwent RRP, the wide VA group had significantly shorter operative times and the complication rate was significantly lower when compared to the narrow VA group. In contrast, in patients who underwent RALP, there was no difference according to the VA.

Operative time is used as an indicator of efficiency and a quality improvement initiative. ${ }^{15,16}$ Therefore, several studies have analyzed predictors that are associated with longer operative times for RALP. ${ }^{16}$ Birkmeyer et al. ${ }^{17}$ reported that higher surgeon volume was related to shorter operative times in robot and open RRP. Zilberman et al. ${ }^{18}$ identified obesity as an independent predictor of longer operative times in RALP patients.

On multivariate analysis for complications, there were no significantly associated factors. The fact that occurrence of complications is very low may be relevant with this result. On the other hand, for operative time, various factors including VA were significantly associated.

In our study, analysis of pelvic anatomy by using preoperative MRI indicated that it was possible to predict perioperative outcomes such as operative time and complication rates, 
as well as long-term operative outcomes such as postoperative incontinence. Therefore, the VA was considered as predictive factors. In addition, these anatomic analyses detected by using preoperative MRI did not reveal any difference in patients who underwent RALP. In this respect, the VA will help to decide the surgical approach. In particular, we have been applying the exasperated Trendelenburg position of over $30^{\circ}$ to patients with a broad VA to secure a wide operative visual field, recently.

This study has some limitations. Our results are based on a relatively small sample size because the study was performed by using a retrospective design at a single institution. In addition, grouping was distributed relatively unequally. Nevertheless, we were able to demonstrate that the operative time could be predicted, and the surgical approach could be decided, simply by referring to preoperative MRI images.

\section{CONCLUSIONS}

The VA observed on midsagittal T2-weighted images could be used to predict the operative time and the occurrence of complication after retropubic RRP, which may indicate the surgical value of retropubic RRP.

\section{CONFLICT OF INTEREST}

The authors claim no conflicts of interest.

\section{REFERENCES}

1. Huang KH, Carter SC, Hu JC. Does robotic prostatectomy meet its promise in the management of prostate cancer? Curr Urol Rep 2013;14:184-91

2. Bill-Axelson A, Holmberg L, Ruutu M, Häggman $M$, Andersson SO, Bratell S, et al. Radical prostatectomy versus watchful waiting in early prostate cancer. N Engl J Med 2005; 352:1977-84

3. Ficarra V, Novara G, Fracalanza S, D'Elia C, Secco S, Iafrate $\mathrm{M}$, et al. A prospective, non-randomized trial comparing robot-assisted laparoscopic and retropubic radical prostatectomy in one European institution. BJU Int 2009;104:534-9

4. Ficarra V, Novara G, Artibani W, Cestari A, Galfano A, Graefen M, et al. Retropubic, laparoscopic, and robot-assisted radical prostatectomy: a systematic review and cumulative analysis of comparative studies. Eur Urol 2009;55:1037-63

5. Gandaglia G, Sammon JD, Chang SL, Choueiri TK, Hu JC,
Karakiewicz PI, et al. Comparative effectiveness of robot-assisted and open radical prostatectomy in the postdissemination era. J Clin Oncol 2014;32:1419-26

6. Menon M, Tewari A, Baize B, Guillonneau B, Vallancien G. Prospective comparison of radical retropubic prostatectomy and robot-assisted anatomic prostatectomy: the Vattikuti Urology Institute experience. Urology 2002;60:864-8

7. Hu JC, Gandaglia G, Karakiewicz PI, Nguyen PL, Trinh QD, Shih YC, et al. Comparative effectiveness of robot-assisted versus open radical prostatectomy cancer control. Eur Urol 2014;66:666-72

8. Diaz M, Peabody JO, Kapoor V, Sammon J, Rogers CG, Stricker $\mathrm{H}$, et al. Oncologic outcomes at 10 years following robotic radical prostatectomy. Eur Urol 2015;67:1168-76

9. Jacobs EF, Boris R, Masterson TA. Advances in robotic- assisted radical prostatectomy over time. Prostate Cancer 2013;2013:902686

10. Dindo D, Demartines N, Clavien PA. Classification of surgical complications: a new proposal with evaluation in a cohort of 6336 patients and results of a survey. Ann Surg 2004;240: 205-13

11. Sohn DW, Hong CK, Chung DJ, Kim SH, Kim SJ, Chung $\mathrm{J}$, et al. Pelvic floor musculature and bladder neck changes before and after continence recovery after radical prostatectomy in pelvic MRI. J Magn Reson Imaging 2014;39:1431-5

12. Lim TJ, Lee JH, Lim JW, Moon SK, Jeon SH, Chang SG. Preoperative factors predictive of continence recovery after radical retropubic prostatectomy. Korean J Urol 2012;53:524-30

13. Matikainen MP, von Bodman CJ, Secin FP, Yunis LH, Vora $\mathrm{K}$, Guillonneau $\mathrm{B}$, et al. The depth of the prostatic apex is an independent predictor of positive apical margins at radical prostatectomy. BJU Int 2010;106:622-6

14. Neill MG, Lockwood GA, McCluskey SA, Fleshner NE. Preoperative evaluation of the "hostile pelvis" in radical prostatectomy with computed tomographic pelvimetry. BJU Int 2007;99:534-8

15. Scales CD Jr, Jones PJ, Eisenstein EL, Preminger GM, Albala DM. Local cost structures and the economics of robot assisted radical prostatectomy. J Urol 2005;174:2323-9

16. Gillespie BM, Chaboyer W, Fairweather N. Factors that influence the expected length of operation: results of a prospective study. BMJ Qual Saf 2012;21:3-12

17. Birkmeyer JD, Stukel TA, Siewers AE, Goodney PP, Wennberg DE, Lucas FL. Surgeon volume and operative mortality in the United States. N Engl J Med 2003;349:2117-27

18. Zilberman DE, Tsivian M, Yong D, Albala DM. Surgical steps that elongate operative time in robot-assisted radical prostatectomy among the obese population. J Endourol 2011; 25:793-6 